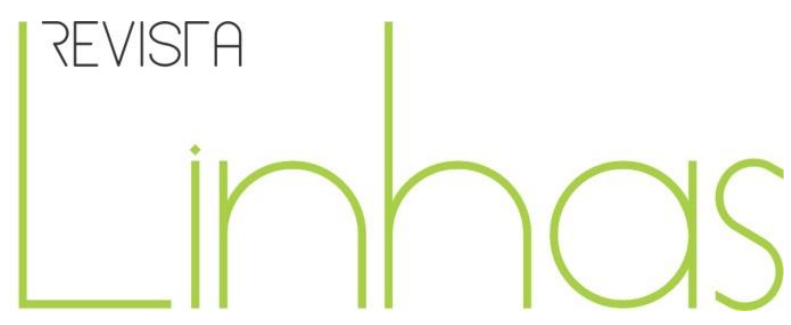

\title{
"Il duro calle". Le memorie di un ispettore scolastico italiano (Berengario Galileo Amorosa - 1865/1937)
}

\begin{abstract}
Resumo
Il contributo propone un caso di studio costituito dalle memorie scolastiche pubblicate nel 1912 dall'ispettore italiano Berengario Galileo Amorosa (intellettuale, storico e studioso), per mettere a fuoco la doppia opportunità che una simile tipologia di fonte offre alla ricerca. Per un verso, le pagine di Amorosa rimandano ad una riflessione di tipo metodologico intorno alla "polivalenza" di questa fonte storiografica, in bilico tra testimonianza e narrazione, segno personale e scrittura pubblica dalla difficili definizione come suggerito da Vinao Frago. Per l'altro, sospingono verso una più circostanziata ricostruzione della "storia della funzione ispettiva" in area italiana, ancora in gran parte da scrivere. L'interesse investe, in questa sede, il genere testuale letterario rappresentato dalle memorie autobiografiche degli ispettori di cui il testo di Amorosa offre un modello significativo. A metà strada tra resoconto e ricordo personale, la conoscenza del mondo scolastico è restituita dai ricordi e dalle storie di un osservatore "speciale": ne viene fuori una rappresentazione della scuola tra fine Ottocento e primordi del Novecento che, oltre a rievocare "le angustie, le miserie e i dolori della classe magistrale", ci restituisce informazioni sulla quotidianità scolastica, attraverso la "rappresentazione dell'ambiente molteplice. Le storie sono affidate ad una cifra rievocativa e narrativa personale che non può naturalmente prescindere dal quadro dell'esperienza collettiva che si colloca sullo sfondo. In tal senso acquista importanza il lavoro di Amorosa che rispecchia "fedelmente quattordici anni di vita vissuta per la scuola elementare, fra genti e paesi diversi", lungo il "duro calle" nei primi decenni del Novecento. Le sue memorie , in definitiva, contribuiscono quale genere testuale, a comprendere in maniera ravvicinata i principali processi di cambiamento educativo da cui fu attraversata la vita scolastica italiana, tra Otto e Novecento.
\end{abstract}

Parole-chiave: Ispettore Scolastico. Storia della Funzione Ispettiva. Vita Scolastica Italiana. Otto e Novecento.

\section{Para citar este artigo:}

D’ALESSIO, Michela. "Il duro calle". Le memorie di un ispettore scolastico italiano (Berengario Galileo Amorosa - 1865/1937). Revista Linhas. Florianópolis, v. 18, n. 36, p. 178-199, jan./abr. 2017.

DOI: $10.5965 / 1984723818362017178$

http://dx.doi.org/10.5965/1984723818362017178

\author{
Michela D'Alessio \\ Universidade de Estudos de \\ Basilicata - Itália \\ michelina.dalessio@unibas.it
}




\title{
"O árduo caminho": Memórias de um inspetor escolar italiano (Berengario Galileo Amorosa, 1865-1937)
}

\begin{abstract}
Resumo
Este artigo propõe um estudo de caso das memórias do serviço escolar publicadas em 1912 pelo inspetor escolar italiano Berengario Galileo Amorosa (intelectual, historiador e erudito), visando focar a dupla oportunidade que fontes similares oferecem à pesquisa. Por um lado, as páginas de Amorosa remetem a uma reflexão metodológica em torno da "versatilidade" dessa fonte historiográfica, pairando entre testemunho e narrativa, marca pessoal e escrita pública de difícil definição, como sugerido por Vinao Frago. Por outro, incitam uma reconstrução mais detalhada da "história da inspetoria" na área italiana, ainda em grande parte a ser escrito. Interessa, aqui, o gênero literário representado pelas memórias autobiográficas de inspetores, do qual o texto de Amorosa oferece um modelo significativo. A meio caminho entre registro e memória pessoal, o conhecimento sobre o mundo escolar é restituído pelas memórias e histórias de um observador "especial": emerge uma representação da escola entre o fim do século XIX e início do século XX que, além de evocar "as angústias, misérias e tristezas da classe magistral", restitui informações sobre o cotidiano escolar por meio da "representação do ambiente complexo". As histórias são confiadas a uma chave evocativa e narrativa pessoal que não podem naturalmente prescindir do âmbito da experiência coletiva que ocorre em segundo plano. Neste sentido, adquire importância o trabalho de Amorosa, que reflete "fielmente catorze anos de vida dedicada ao ensino fundamental, entre povos e cidades distintos", no decorrer do "árduo caminho" nas primeiras décadas do século XX. Suas memórias, em última análise, contribuem enquanto gênero textual para a compreensão aproximada dos principais processos de mudança educacional que atravessaram a vida escolar italiana entre os séculos XIX e XX.
\end{abstract}

Palavras-chave: Inspetor Escolar. História da Inspetoria. Vida Escolar Italiana. Séculos XIX e XX. 


\title{
"The hard road." The memories of an Italian school inspector (Berengario Galileo Amorosa - 1865/1937)
}

\begin{abstract}
This paper proposes a case study constituted by school memories published in 1912 by the Italian inspector Berengario Galileo Amorosa (intellectual, historian and scholar), with the objective of calling attention to the double opportunity that a similar kind of source offers to the research. On the one hand, the pages of Amorosa send us back to methodological reflections on the "versatility" of this historiographical source, poised between testimony and narrative, personal mark and public writing, of difficult definition, as suggested by Vinao Frago. On the other hand, they impel towards a more detailed reconstruction of the "history of the inspection function" in the Italian area, still largely to be written. At this point, the interest is directed to the literary text genre represented by the autobiographical memories of the inspectors, from which the text of Amorosa offers a significant example. Halfway between report and personal remembrance, the knowledge about the school world is restored from the memories and stories of a "special" observer: what emerges is a depiction of the school in the late nineteenth and the beginning of the twentieth century, which, apart from recalling "the distress, the miseries and sorrows of the class of teachers", gives us information about the everyday school life, through representation of this manifold environment. The stories are entrusted to a recollection character and personal narrative that naturally cannot renounce the framework of collective experience that is placed in the background. In this sense, the work of Amorosa gains importance, which "faithfully reflects fourteen years of life dedicated to the elementary school in different countries and among different peoples", along the "hard road" in the early decades of the twentieth century. Ultimately, his memoirs, as text genre, contribute in a very close way for the understanding of the major processes of educational change experienced by the Italian school life in the nineteenth and twentieth centuries.
\end{abstract}

Keywords: Scholar Inspector. History of the Inspection Function. Italian School Life. Nineteenth and Twentieth Centuries. 
Tu proverai sì come sa di sale Lo pane altrui, e com'è duro calle Lo scendere e il salir per l'altrui scale

(Dante, Paradiso, XVII)

\section{Premessa: un caso di studio italiano sulla ispezione scolastica}

Il contributo propone il caso di studio offerto dalle memorie scolastiche pubblicate nel 1912 dall'ispettore italiano Berengario Galileo Amorosa ${ }^{1}$, allo scopo di mettere a fuoco le principali opportunità che una simile tipologia di fonte assicura alla ricerca. Lo sguardo incentrato sulle vicende biografiche e sulla produzione di segno "scolastico" dell'autorevole esponente del Molise convergeranno verso una disamina specifica della sua memorialistica autobiografica che viene a qualificarsi nella sua esemplarità, tanto per gli aspetti di riflessione storiografica e metodologica che sollecita, quanto per le ravvicinate possibilità di ricostruzione di uno spaccato della funzione ispettiva italiana, tra Otto e Novecento.

\section{La fonte di studio e le opportunità conoscitive: gli ego-documenti e la funzione ispettiva italiana}

Per un verso, le pagine di Amorosa inducono ad una riflessione di tipo metodologico intorno alla "polivalenza" di questa fonte storiografica, a metà strada tra testimonianza e narrazione, su cui si tornerà a breve. Una scrittura che se si presenta autoreferenziale, nel senso che il soggetto parla di sé, è allo stesso tempo incentrata sul racconto delle relazioni stabilite dall'ispettore con i luoghi ed i protagonisti del mondo della scuola che ci racconta, secondo naturalmente il suo sguardo e quindi un modello

\footnotetext{
${ }^{1}$ B.G. Amorosa, II "duro calle". Memorie d'un ispettore scolastico, Milano Roma - Napoli, Società editrice Dante Alighieri di Albrighi \& C., 1912. Una sintesi dell'indagine condotta intorno alla figura ispettiva dell'Amorosa è stata inizialmente presentata in M. D'Alessio, "Il duro calle". Memorie di un ispettore scolastico (Berengario Galileo Amorosa, 1865-1937), in C. Yanes, J. Meda (a cura di), International Symposium, School memories. New trends in Historical research into Education Heuristic Perspectives and Methodological issues (Siviglia, 22-23 settembre 2015). Abstracts, p. 22.
} 
personale di rappresentazione. A tale riguardo si rinvia, in particolare, alla difficoltà di definizione di tali fonti ${ }^{2}$ esposta con persuasione da Vinao Frago 3 .

Per l'altro, sospingono verso la ricostruzione della "storia della funzione ispettiva" in area italiana ${ }^{4}$. Un primo contributo in tale direzione è provenuto negli anni "6o da Giacomo Cives con la riproposizione di alcuni documenti e testimonianze di ispettori, visitatori ed educatori ${ }^{5}$. Lo studioso ha attribuito gli elementi di criticità della tradizione ispettiva italiana alla confusione dei suoi compiti e all'indistinto suo campo di azione, oscillante tra la funzione tecnico-didattica e quella più propriamente amministrativa. Visitatori, osservatori delle vicende delle tante scuole, gli ispettori hanno contribuito alla conoscenza concreta delle diverse situazioni in cui si è fatta scuola in Italia. Le loro relazioni, non adeguatamente studiate dalla storiografia scolastico-educativa, restituiscono informazioni non secondarie sui loro "viaggi pedagogici". Spesso non solo documenti di natura burocratica e amministrativa, le visite ispettive ci introducono alla conoscenza di fatti e situazioni minute e concrete sul piano delle applicazioni pratiche delle direttive pedagogico-didattiche in aiuto ai maestri e della registrazione di indagini ambientali e sociologiche.

Su questa linea si colloca lo stesso interesse per l'esperienza della personalità ritenuta "più autenticamente ispettiva per costituzione e temperamento", quella di Giuseppe Lombardo Radice ${ }^{6}$. Il lavoro con cui mi sono interessata alla ricostruzione di un

\footnotetext{
${ }^{2}$ Sul tema degli "ego-documenti" rinvenibili nelle scritture magistrali - e qui ispettive - si rinvia alle considerazioni esposte intorno specificamente ai registri scolastici in M. D'Alessio, Life at school: class registers as a new source of studying historical and educational heritage, in A.M. Badanelli Rubio, M. Poveda Sanza, C. Rodriguez Guerrero (a cura di), Pedagogia museistica. Practicas, usos didacticos e investigacion del patrimonio educativo, Atti della VI Jornadas cientifica della Sociedad Espanola para el Estudio del Patrimonio Historico educativo SEPHE (Madrid, 22-24 ottobre 214), Madrid, Universidad Complutense de Madrid, Facultad de Educacion, 2014, pp. 401-409.

${ }^{3} \mathrm{Cfr}$. A. Vinao Frago, Relatos y relaciones autobiograficas de profesores y maestros, in A. Escolano Benito, J.M. Hernandez Diaz (a cura di), La memoria y el deseo. Cultura de la escuela y educacion deseada, Valencia, Tirant Lo Blanch, 2002, pp. 135-175.

${ }^{4}$ Sulle trasformazioni dell'istituto ispettivo scolastico si rimanda al lavoro di G. Decollanz, La funzione ispettiva dalla legge Casati ad oggi, Roma, Armando, 1984, lavoro a cui, come è stato rimarcato da più parti, non ha fatto seguito una ricostruzione tematica dei singoli profili e caratteristiche dell'intero corpo ispettivo.

${ }^{5} \mathrm{G}$. Cives, Cento anni di vita scolastica in Italia. Ispezioni e inchieste da Gino Capponi a Giuseppe Lombardo Radice, Roma, Armando Editore, 1960 e Id., Cento anni di vita scolastica in Italia. Ispezioni e inchieste dall'idealisno a oggi negli scritti di G. Lombardo Radice, G. Isnardi, G. Giovinazzi, F. Betttini, A. Marcucci, L. Volpicelli, L. Borghi, A. Visalberghi, ivi, 1967.

${ }^{6}$ Non è stato sempre posto in sufficiente rilievo il collegamento tra l'ampio impegno educativo del
} 
quadro efficace, poiché proveniente da una prospettiva coeva, dell'applicazione della riforma scolastica del 1923 nelle diverse circoscrizioni scolastiche del Paese, attinge a tale rinnovata tipologia di fonti per la scuola risalendo, tra i documenti che, in particolare, Lombardo Radice considerava "ragguardevoli della storia della scuola italiana nel nostro tempo"7 per l'appunto alle "relazioni annue che usano scrivere gli ispettori scolastici"”. Addentrarsi “nelle modalità del 'fare scuola' attrae, infatti, oggi più di ieri lo sguardo degli storici dell'educazione poiché rappresenta la maniera per penetrare dall'interno nell'accertamento circostanziato dei fattori, dei meccanismi, dello scarto e delle diverse dinamiche attraverso cui le disposizioni ministeriali e i progetti pedagogici hanno trovato trasmissione in un reticolo fatto di maglie più larghe, ma anche più fitte, fino $a$ raggiungere le singole realtà scolastiche disseminate nel territorio nazionale" ${ }^{9}$.

Stimoli più recenti sono stati ripresi da Giorgio Chiosso, che si è soffermato, oltre che sul ruolo degli ispettori scolastici nella vigilanza del rispetto delle leggi e dei regolamenti, così come nella diffusione dei nuovi metodi didattici, sulla necessità di una sistematica rassegna degli ispettori in Italia ${ }^{10}$. Lo studioso lamenta, infatti, l'esigua disponibilità di informazioni sul corpo complessivo degli ispettori scolastici italiani in rapporto alle sistematiche rassegne esistenti, ad esempio, in ambito francese, e la necessità al riguardo di una più complessiva ricognizione archivistica. Osservazioni in tale direzione, insieme a considerazioni puntuali sulle caratteristiche e gli orientamenti del corpo ispettivo negli anni della Destra storica sono stati di recente utilmente proposti dal documentato lavoro di Alberto Barausse intorno alle indagini di Bargoni e Bonghi sulla questione dei libri di

\footnotetext{
pedagogista siciliano con la sua funzione di "ispettore nato": infatti, "che fosse consigliere scolastico dell'Associazione del Mezzogiorno, o direttore generale, visitatore non ufficiale o docente universitario, sapeva scoprire le scuole più modeste e umili in cui si faceva qualcosa di buono allo stesso modo di come studiava quelle più affermate e rilevanti, per diffonderne le trovate, $i$ frutti, $i$ successi; sapeva sostenere e aiutare ciascun insegnante, anche nella situazione più diversa o difficile o singolare". Ivi, p. 30.

${ }^{7}$ G. Lombardo Radice, Presentazione a R. Dal Piaz, Esperienze didattiche di un ispettore trentino: relazionestudio sulle scuole della circoscrizione di Trento (1925-1926), Roma, Associazione per il Mezzogiorno, 1928, p. 3.

${ }^{8}$ L'interesse per le "esperienze didattiche" degli ispettori scolastici costituisce un fattore cospicuo della dimensione storiografica a cui guarda il mio lavoro sulla manualistica regionale introdotta dopo la riforma Gentile del sistema scolastico italiano: cfr. M. D'Alessio, A scuola fra casa e patria. Dialetto e cultura regionale nei libri di testo durante il fascismo, Collana Biblioteca del "Centro di documentazione e ricerca sulla storia delle istituzioni scolastiche, del libro scolastico e della letteratura per l'infanzia" dell'Università degli Studi del Molise, Lecce, Pensa Multimedia, 2013.

${ }^{9}$ Ivi, p. 38.

${ }^{10} \mathrm{G}$. Chiosso, Alfabeti d'Italia. La lotta contro l'ignoranza nell'Italia unita, Torino, SEI, 2011, pp. 199-203.
} 
testo durante i primi quarant'anni postunitari" ${ }^{11}$. Alcuni fasci di attenzione intorno alla stampa tecnica specializzata negli anni tra idealismo e fascismo sono stati inoltre rivolti dal contributo che ha gettato luce sull'esperienza della rivista professionale «La Tecnica scolastica», diretta da Alfredo Saraz ${ }^{12}$.

L'interesse, tuttavia, non investe in maniera prevalente in questa sede le specifiche relazioni delle visite ispettive - pur non adeguatamente studiate dalla storiografia scolastico-educativa quali scritture istituzionali, sempre in bilico tra funzione burocratica e tecnico-didattica. Riguarda, invece, principalmente il genere testuale letterario rappresentato dalle memorie autobiografiche degli ispettori di cui il testo di Amorosa offre un modello significativo.

\section{Il profilo biografico e dell'“uomo di scuola"}

\footnotetext{
11 "Le relazioni aiutano, così, anche ad entrare nei meccanismi di una professione particolarmente gravosa e impegnativa. In realtà poco conosciamo della identità e della realtà di coloro che parteciparono alla funzione ispettiva durante gli anni della destra storica. Non esistono, purtroppo, studi sistematici che offrano un panorama esaustivo delle caratteristiche del corpo ispettivo italiano, componente significativa della più generale nuova amministrazione scolastica italiana. Individuarne alcune può, però, aiutare a comprendere meglio i differenti e disomogenei approcci dei funzionari periferici, sulla questione dei libri di testo": cfr. A. Barausse, Nonostante tanto diluvio di libri scolastici. I libri di testo per le scuole elementari e le indagini ministeriali di Bargoni e Bonghi durante gli anni della Destra storica (1869-1875), Lecce, Pensa Multimedia, 2015, p. 20. Riferimenti utili intorno alla formazione del corpo ispettivo in epoca giolittiana sono presenti già in A. Barausse, I maestri all'università: la Scuola pedagogica di Roma 1904-1923, Perugia, Morlacchi, 2004, passim.

${ }^{12}$ Su questa specifica stampa specializzata professionale che trovò nella «Tecnica scolastica» un "organo sereno ed autorevole degli uomini di scuola" e sotto la guida del suo attivo direttore Alfredo Saraz divenne una singolare "officina" educativa ed amministrativa durante le sue cinque annate di vita dal 1923 al 1927, cfr. A. Barausse, M. D’Alessio, "La salvezza della scuola verrà dai tecnici”. La stampa pedagogica in Italia tra idealismo e fascismo: la «Tecnica scolastica» (1923-1927), in J.M.H. Diaz (a cura di), La prensa de los escolares y estudiantes. Su contribucion al patrimonio historico educativo, Salamanca, Ediciones Universidad de Salamanca (Collecion Aquilafuente, 210), 2015, pp. 611-626. La rivista mostra di seguire fin dal suo apparire un programma ben definito, nell'occuparsi "di didattica e di amministrazione scolastica per ogni ordine e grado di scuola" e nel presentarsi allo stesso tempo quale "organo di consultazione, di studio, di incitamento verso il meglio del nostro mondo scolastico". I principali collaboratori del comitato redazionale provengono dal mondo dei direttori e degli ispettori scolastici. Alcune figure hanno ricoperto ruoli importanti nell'amministrazione scolastica centrale o periferica o all'estero. Tra di essi insegnanti e poi direttori o ispettori come Giovanni Di Giusto, Libero Forte, Amedeo Cominetti, Giacomo Carenzi, Evaristo Marsili, Michele Angelastri. A questi vanno ad aggiungersi i profili più vicini alla personalità ed al neoidealismo di Lombardo Radice, tra cui si distinguono gli ispettori scolastici Riccardo Dal Piaz, Giovanni Lucaroni, Edoardo Predome, insieme a firme occasionali di maggior prestigio, Vidari, Giovanazzi, Tauro ed altre invece espresse dal mondo magistrale femminile, tra cui Amalia Paluffo. La rivista passò da un appoggio incondizionato alla "rivoluzione scolastica in marcia" della riforma avviata da Gentile, ad una posizione maggiormente critica negli ultimi anni, riflettendo nel complesso l'eterogeneità delle diverse componenti del fascismo scolastico e pedagogico italiano. Ibidem.
} 
Per meglio conoscere i tratti della biografia istituzionale di intellettuale, storico e studioso dalla impegnata carriera scolastica e ministeriale, è utile ripercorrere la stessa Autobiografia dell'autore presente tra gli scritti In memoria del Gr.Uff. Berengario Galileo Amorosa Provveditore agli studi ${ }^{13}$. Avviato agli studi elementari dal padre, educatore benemerito della comunità riccese ${ }^{14}$, Berengario proseguì gli studi ginnasiali in vari convitti, prima ad Assisi nel 1875 e poi dal 1879 a Napoli, dove l'anno successivo conseguì la licenza ginnasiale. Tornò a studiare nel prestigioso convitto "Mario Pagano" di Campobasso $^{15}$ nel 1880 , ottenne quindi la licenza liceale a Napoli, nel 1884-85, oltre alla patente elementare di grado superiore presso la scuola normale partenopea. Subito dopo, insegnò per cinque anni nel suo paese d'origine ${ }^{16}$ e in seguito fu inviato a Montenero di Bisaccia, fino al 1891.

Nel febbraio di quell'anno, infatti, l'Amorosa risultò vincitore del concorso a ispettore scolastico, con destinazione Conegliano Veneto. Prese servizio nel comune del trevigiano il 27 marzo del 1891, restandovi per un anno. È noto che proprio la raggiunta unità politica e amministrativa promuoveva numerosi trasferimenti dicf ufficiali e impiegati dello Stato, tra cui principalmente insegnanti, nel nuovo raggio di una società nazionale in cui andava a favorirsi una circolazione delle persone e delle idee più ampia che per il passato. Secondo pertanto questa diffusa mobilità professionale e didattica del

\footnotetext{
${ }^{13}$ B.G. Amorosa, Autobiografia, in In memoria del G. Uff. Dottor Berengario Galileo Amorosa Provveditore agli studi, MDCCCLIV-MCMXXXVII, s.n.t. [1938], pp. 11-23. Di recente il profilo dell'Amorosa è stato proposto da M. D'Alessio, ad vocem nel repertorio del DBE Dizionario Biografico dell'Educazione. 1800-2000, diretto da Giorgio Chiosso e Roberto Sani, Milano, Editrice Bibliografica, 2013, 2 voll., v. I, scheda n. 75, pp. 45-46. Una proficua rassegna di contributi sulla personalità e del ruolo dell'Amorosa era provenuta dal volume di $G$. Palmieri, A. Santoriello (a cura di), Berengario Galileo Amorosa. Atti del Convegno, Riccia, 18 luglio 1987, Riccia, Associazione Culturale "Pasquale Vignola", 1989. I primi risultati di studio sono stati proposti in M. D’Alessio, Scuola e lingua nel Molise di fine Ottocento, Napoli, Edizioni Scientifiche Italiane, 2005, pp. 130-166. Si veda ora G. Palmieri, Berengario Galileo Amorosa. L'intellettuale e lo storico, in Id., Studi di storiografia molisana, Campobasso, Palladino Editore, 2016, pp. 89-105.

${ }^{14}$ Cfr. A. Santoriello, Berengario Galileo Amorosa, in Id. (a cura di), Riccia tra biografie e storia. Ricerche su personaggi illustri dell'Ottocento e del Novecento, Riccia, Associazione culturale "Pasquale Vignola", 1997, pp.196-197 e Id., I fratelli Amorosa, ivi, pp. 125-136.

${ }^{15}$ Sul blasonato ginnasio-liceo del capoluogo molisano si rinvia alla ricostruzione prospettata nel volume $\mathrm{M}$. D'Alessio, Vita tra i banchi nell'Italia meridionale. Culture scolastiche in Molise fra Otto e Novecento, premessa di Alberto Barausse, Collana Biblioteca del "Centro di documentazione e ricerca sulla storia delle istituzioni scolastiche, del libro scolastico e della letteratura per l'infanzia" dell'Università degli Studi del Molise, Campobasso, Palladino Editore, 2011, pp. 13-44.

${ }^{16}$ Dal 1 novembre 1884 al 31 ottobre 1889 . I documenti relativi ai primi anni d'insegnamento dell'Amorosa sono conservati nell'archivio storico comunale di Riccia: Berengario Galileo Amorosa, in Istruzione pubblica: maestri (1806-1896), b. 110, f. 761.
} 
tempo, il molisano fu a Pieve di Cadore (12 gennaio 1892-30 settembre 1893), a Sulmona (1 ottobre 1893-6 novembre 1895), a Penne, sempre in Abruzzo (dal 7 novembre 1895 al 10 novembre 1897); quindi in Sardegna, a Sassari (11 novembre 1897-13 aprile 1898) e per un

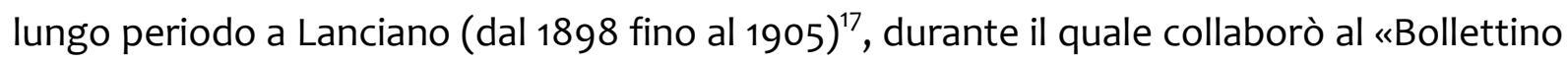
magistrale» locale con articoli “d’indole didattica e di politica scolastica”.

Amorosa parla proprio nella prefazione alle sue memorie della ridotta remunerazione economica riconosciuta ad un ispettore al suo primo incarico, con 1500 lire di stipendio e del più arduo cammino che gli si presentava per fare piccoli progressi nella carriera, fortemente condizionati dall'esiguo numero dell'organico previsto all'epoca $^{18}$, fino al passaggio al ruolo di Provveditore dopo circa quindici anni di peregrinazioni per tutto lo stivale italiano, giungendo a guadagnare 2000 lire.

Oratore eloquente, storico, letterato e scrittore, Amorosa unì la personalità di intellettuale incline allo studio e al multiforme impegno culturale ai tratti maggiori di figura eminente del mondo scolastico. Nel 1903 vinse il concorso bandito in quell'anno per otto provveditori agli studi e dopo aver rinunciato alla sede di Caltanissetta, fu a Catanzaro dal 1905 al 1908. Tornò in Abruzzo restando a Chieti per cinque anni, dal 1908 al 1913, e di lì passò per tre anni a Caserta, fino al 1919. Fu poi chiamato alla direzione generale delle scuole italiane all'estero, ma rinunciò per motivi familiari all'incarico nelle scuole dell'America latina (restando al Ministero fino al 1920). Ottenne nuovamente l'incarico di provveditore a Chieti, dove rimase fino al 30 giugno del 1923. Fu collocato a riposo a 58 anni nel dicembre del 1923, dopo essere stato chiamato a far parte della Commissione centrale per la revisione dei libri di testo presieduta nella prima tornata dallo stesso Giuseppe Lombardo Radice.

\footnotetext{
${ }^{17}$ Su questi anni si rinvia a G. Palmieri, Il contributo della «Rivista abruzzese agli studi del Molise», in «Rivista storica del Sannio», a. VIII (2000), n. 13, pp. 305-323.

${ }^{18}$ II R.D. 29 settembre 1885 prevedeva un organico, restato in vita per dieci anni, di 25 ispettori in prima classe, 35 in seconda, 87 in terza, 91 in quarta. La retribuzione nella classe più alta era di 3000 lire. II R.D. 8 agosto 1895 ridusse gli ispettori a 218 formando tre classi. Un'utile raccolta delle disposizioni sul "servizio ispettivo" fino agli anni Venti del "900 è in G Carenzi, Manuale di legislazione scolastica, Torino, Paravia, 1919 (il secondo volume è del 1920), specie al secondo capitolo. Per una trattazione completa degli aspetti riguardanti "il soggetto della funzione di vigilanza scolastica" secondo la legislazione italiana e con riferimenti a quella di alcuni Stati esteri, si segnalano due specifici lavori, molto ben documentati e tra loro complementari: cfr. A. Saraz, La tecnica dell'ispezione scolastica, Torino, G.B. Paravia \& C., 1921 e Id., Vicende storiche ed attuale ordinamento della Direzione ed Ispezione scolastica, Torino, G.B. Paravia \& C., 1922.
} 
L'ampia produzione dell'Amorosa spazia da quella iniziale di segno poetico ${ }^{19}$ a quella successiva pubblicistica, storica ed erudita in prosa interessata al materiale demologico sulle tradizioni molisane ${ }^{20}$, fino a quella destinata espressamente alla scuola, secondo una poliedricità di segno culturale e stilistico.

\section{La produzione di segno scolastico}

L'incidenza della componente scolastica è attestata dalla varietà e continuità degli scritti dedicati dall'Amorosa alla trattazione di tale tematica. Questi comprendono i primi articoli apparsi in importanti riviste pedagogiche nazionali, tra cui l'«Avvenire educativo», il «Rinnovamento scolastico» e il «Risveglio educativo». Vi rientrano poi i racconti sul mondo scolastico ed i suoi principali protagonisti, ispirati dalle diverse esperienze vissute nei molti luoghi con cui l'autore venne in contatto e raccolti sotto la forma narrativa breve del medaglione biografico, del saggio e del bozzetto nel volume Paria moderno. Documenti umani ${ }^{21}$, in cui sono riportati sulla pagina storie vere di vita, di tanti maestri e maestre "paria", vittime di discriminazioni e ingiustizie sociali nell’'talia postunitaria. Su questa stessa cifra narrativa e rievocativa personale, come si avrà meglio modo di illustrare, i ricordi de Il duro calle. Memorie d'un ispettore scolastico, dati alle stampe nel 1912, propongono ancora l'autore quale narratore esterno e allo stesso tempo personaggio interno del mondo che racconta. Gli interventi dedicati specificamente alle questioni scolastiche e pedagogiche dibattute nei primi vent'anni del Novecento sono raccolti nelle Note didattiche e di politica scolastica pubblicati nel $1903^{22}$ e nel volume successivo al primo conflitto mondiale La scuola in provincia di Terra di Lavoro nei primi tre anni di guerra, del $1918^{23}$.

Accanto a tali lavori, un posto a sé - per la originale tipologia testuale ed i contenuti precipui di cultura regionale - occupano i due principali volumi di specifica destinazione

\footnotetext{
${ }^{19}$ B.G. Amorosa, Epodon: versi, Conegliano, Tip. F. Cagnani, 1891; Id., Cadore. Impressioni liriche, Pieve di Cadore, E. Berengan e C., 1893.

${ }^{20}$ Id., Riccia nella storia e nel folklore, Casalbordino, Tip. N. De Arcangelis, 1903.

${ }^{21}$ Palermo, Sandron, 1895.

${ }^{22}$ Saggi critici, Lanciano, Tip. A. Rosati, 1903.

${ }^{23}$ Note ed appunti del R. Provveditore agli Studi Dott. B.G. Amorosa, Caserta, Tipografia Economica B. De Simone, 1918.
} 
scolastica per il Molise e l'Abruzzo, che furono pubblicati dall'Amorosa tra il 1924 ed il 1925 all'interno della prestigiosa collezione Mondadori degli almanacchi regionali ${ }^{24}$. Nel contesto della rinnovata produzione editoriale rivolta allo studio della cultura regionale secondo il dettato dei programmi lombardiani, i due sussidiari si connotarono in senso segnatamente antropo-geografico rilasciando un ampio spazio agli usi locali, ai costumi, alla poesia dialettale, ai rituali tipici, accanto all'accumulo dei materiali eruditi cari all'autore. Ritorna in queste pagine quella sorta di compromesso rinvenibile nella pratica scrittoria dell'Amorosa, tra la tendenza all'invenzione e le ricerche storico-erudite, una tensione contrapposta tra la disponibilità alla scrittura in sé letteraria e l'inclinazione all’osservazione diretta della realtà sociale, scolastica o folklorica ${ }^{25}$.

\section{Le scritture ispettive: un trascurato fronte investigativo}

Provando a ripercorrere nella parabola personale del funzionario italiano il fronte investigativo, che si segnala per essere stato solo marginalmente considerato dalla storiografia del settore, sarà utile classificare le diverse tipologie testuali isolabili nel novero complessivo delle scritture ispettive. Preme ribadire come il tratto preminente che in questa sede si intende enfatizzare prendendo le mosse proprio dall'esperienza delle memorie autobiografiche del molisano è il piano delle rappresentazioni del sé in relazione con il mondo della scuola, così come emerge da diversificati luoghi testuali. Soffermandosi, pertanto, sui lavori provenienti dalla penna degli ispettori operanti nelle diverse zone della "Italia delle Italie", si proverà ad enucleare quelli principali.

Nel primo corpus testuale si collocano i verbali e relazioni delle visite ispettive che offrono una osservazione strutturata sull'andamento delle scuole in un dato luogo ed in

\footnotetext{
${ }^{24}$ B.G. Amorosa, II Molise. Almanacco regionale, Mondadori, 1924, poi ristampato con saggio introduttivo di Giulio Di Iorio, dall’Associazione Culturale “P. Vignola”, Riccia, nel 1990. Di lorio esamina come "le sollecitazioni maggiori a scrivere un libro di cultura regionale, provengono da quegli intendimenti didattici interni all'Amorosa - per professione ai vertici dell'ordinamento scolastico - sempre attento sia alle problematiche della scuola, sia a quelle di chi in essa quotidianamente opera. Ed ai maestri di scuola l'Amorosa aveva dedicato una raccolta di brevi racconti nei quali aveva descritto, seppure con molta enfasi, il loro difficile e duro lavoro quasi sempre invisibile agli altri" (pp. XIX-XX). Amorosa l'anno successivo si vide approvato anche l'almanacco per la regione dell'Abruzzo. Riferimenti ai due almanacchi approvati dell'Amorosa, posti in confronto specialmente con l'altro licenziato da Eugenio Cirese sempre per il Molise, sono contenuti in A. Barausse, M. D’Alessio, "Dalla piccola alla grande patria". Libri dialettali e almanacchi regionali per la scuola elementare, in G. Chiosso (a cura di), TESEO ‘90o. Editori scolastico-educativi del primo Novecento, Milano, Editrice Bibliografica, 2008, specie a p. XLIII.

${ }^{25}$ Ampi approfondimenti in M. D’Alessio, A scuola fra casa e patria, cit., pp. 133-137.
} 
un dato tempo. Si pensi in tale cerchio all'importanza dell'attività di vigilanza e controllo che venne svolta dal personale dell'Associazione Nazionale per gli Interessi del Mezzogiorno d'Italia, nelle regioni per le quali durante il fascismo ottenne la delega per la lotta contro l'analfabetismo ${ }^{26}$.

Subito dopo figurano i diari, le autobiografie, le memorie. Accanto al "duro calle" di Amorosa non sono da trascurare una serie di altri scritti di funzionari centrali e periferici che conobbero gli onori della stampa. È il caso, a titolo esemplificativo, dell'ispettore lucano Antonio Renzi che insieme ad altri lavori sulla storia, la geografia e la cultura regionale, consegnò a fine carriera un'interessante memorialistica intorno a quella che definisce, fin dal titolo, la sua opera di “apostolato" ${ }^{27}$, opera molto affine nei toni e nelle vicende narrate alla esposizione dell'ispettore molisano. Lo stesso Renzi, evidentemente ben introdotto negli ambienti istituzionali e redazionali del tempo, è la firma ricorrente della rubrica "Ispezionando" rintracciata su la «Tecnica scolastica»" ${ }^{28}$, ricadente nel terzo spazio testuale individuato, quello degli articoli e della saggistica di natura tecnica.

Qui si collocano anche le diverse monografie accolte in una specifica Collana di testi di ispettori e vice-ispettori d'argomento pedagogico e didattico-educativo. La “Biblioteca degli ispettori scolastici” fu data alle stampe nel 1912 dall'editore Antonio Vallardi il quale, nel presentarla, specificava come questa comprendesse "gli scritti degli Ispettori e dei Vice-ispettori, senza pregiudiziali pedagogiche o didattiche o filosofiche o scientifiche o sociali. Essa accoglie, perciò, con assoluta obiettività e imparzialità, le manifestazioni delle più disparate scuole o teorie o tendenze, mettendo a solo limite l'organicità dei lavori che gli egregi Autori propongono". I lavori rintracciati coprono un arco temporale di oltre dieci anni (dal 1912 al 1923), attestandosi nel periodo principale di passaggio dall'idealismo al fascismo e documentano un interesse continuativo e sistematico per l'esercizio della funzione tecnica ed educativa ispettiva sviluppato nei

\footnotetext{
${ }^{26}$ Cfr. M. D'Alessio, Our Schools". The work of the Association of Southern Italy against illiteracy in Basilicata (1921-1928), in «History of Education \& Children's Literature», a. X, n. 2 (2015), pp. $463-480$ ed Ead., Igiene e scuole rurali. Itinerari ed esperienze dell'ANIMI in Basilicata durante il fascismo tra educazione e propaganda, in «Rivista di Storia dell'Educazione», n. 2 (2016), in corso di stampa.

${ }^{27}$ A. Renzi, "Apostolato". Memorie autobiografiche di Antonio Renzi, Regio Ispettore scolastico a riposo, Minerbio, Tip. Bevilacqua, [1930].

${ }^{28}$ A. Barausse, M. D’Alessio, "La salvezza della scuola verrà dai tecnici”. La stampa pedagogica in Italia tra idealismo e fascismo: la «Tecnica scolastica (1923-1927), cit.
} 
sedici volumi apparsi per i tipi vallardiani ${ }^{29}$. La quarta tipologia individuata è quella su cui pure, sulla scorta delle pagine di Paria moderno di Amorosa si è fatto già cenno, dei racconti, delle novelle, dei romanzi e dei libri scolastici ${ }^{30}$.

In tutti i testi, esemplificativi dei diversi piani di lavoro professionale e letterario degli ispettori italiani, preme porre in risalto la mobilità del punto di vista degli autori Si procede da quella che possiamo definire osservazione oggettiva, funzionale al compito di "riferire" con puntualità nello svolgimento dell'attività di vigilanza e controllo; alla divergente "soggettività" rinvenibile nella esigenza di "raccontare" le esperienze di vita in particolare svolte mostrando tutta la vicinanza alla classe dei maestri; al ruolo di "mediazione" condotto nelle fasi tese a "istruire" esercitando la specifica funzione tecnica in vista della formazione della coscienza professionale; e, per concludere, alla specifica vena narrativa, rintracciabile nella rielaborazione letteraria dei casi concreti di cronaca, anche essi miranti ad educare tutti gli operatori della vita scolastica italiana alla pubblica coscienza scolastica.

È pertanto possibile seguire la dilatazione dello sguardo dei funzionari ministeriali che procede dal sé del narratore, alla coscienza di appartenenza alla categoria professionale, al mondo della scuola e dei maestri, fino alla società e di lì alla storia.

\section{5. «ll duro calle» degli ispettori: l'impegno "a far maggiormente amare la scuola, i maestri e gl'ispettori"}

\footnotetext{
${ }^{29}$ La collana prese avvio con alcuni "consigli e norme didattiche" rivolti ai giovani insegnanti esordienti e proseguì, oltre che con lavori improntati a questa linea di suggerimenti pratici dati dagli ispettori, con volumi di "punzecchiature e spunti scolastici" e pagine più propriamente pedagogiche fino agli scritti educativi e "di arte didattica". Cfr. D. Borghese, Lettere a una giovine normalista: (dalle rive della Dora), Milano, A. Vallardi, 1912, Biblioteca degli ispettori scolastici 1; G. Antonietti, Dal taccuino di un ispettore: noterelle didattiche, ivi, 1913, 2; F. Esposito, La suggestione come mezzo educativo, ivi, 1914, 3; V. Norscia, Nel campo della pedagogia, ivi, 1914, 4; G. Simeoni, Consigli e norme didattiche agli insegnanti esordienti, ivi, 1915, 5; A. Piccioni, Punzecchiature e spunti scolastici, ivi, 1915, 6; P. Carlini, Del carattere come formazione psicologica: con l'appendice di una conferenza didattica e due discorsi, ivi, 1915, 7; D. De Murtas, L'emozione estetica considerata come mezzo di educazione: note di fisio-psicologia, ivi, 1915, 8; P. Cavazzuti, Il patronato scolastico e le più importanti istituzioni ausiliarie della scuola in Italia, ivi, 1916, 9; I. Dossi, Pagine di pedagogia di un educatore trentino, ivi, 1919, 10; F. Bianchi, Aristide Gabelli nella filosofia positiva e nella pedagogia applicata, ivi, 1920, 12; R. Mariani, Lettere didattiche ad un'esordiente di scuola rurale, ivi, 1920, 13; A. Bianchessi, Scritti educativi, ivi, 1920, 14; A.F. Cossu, Note e riflessi di arte didattica, ivi, 1923, 16.

${ }^{30}$ Sulla riscoperta dell'opera scolastica su menzionata cfr. M. D'Alessio, Scuola e lingua nel Molise di fine Ottocento, cit., specie alle pp. 142-166.
} 
Le storie dell'ispettore molisano sono narrativamente organizzate in un corposo volume di circa 300 pagine e distribuite in 31 capitoli che si snodano dai ricordi iniziali della propria famiglia alle diverse tappe del suo percorso di formazione, fino alla rievocazione dei primi incarichi ispettivi dal Veneto fino all'Abruzzo.

Il ritmo del racconto è affidato ad una cifra rievocativa e narrativa personale che non può naturalmente prescindere dal quadro dell'esperienza collettiva che si colloca sullo sfondo, lasciando cogliere singoli contesti locali nello spettro allargato della scuola nazionale. In tal senso acquista importanza il lavoro di Amorosa che rispecchia fedelmente

quattordici anni di vita vissuta per la scuola elementare, fra genti e paesi diversi, viaggiando d'inverno e d'estate, affrontando sacrifici e disagi, lottando bene spesso contro la collera degli elementi e costantemente contro l'apatia o l'avversione degli uomini $i^{31}$.

Il libro offre un'accurata rappresentazione dell'“ambiente molteplice" con cui l'ispettore era venuto in contatto lungo il suo peregrinare nelle diverse sedi lavorative. Così, alla ricostruzione della personale traiettoria esistenziale e professionale si associa la descrizione di una spiccata capacità di stabilire rapporti fecondi e di interagire “intensamente con tutte le località nelle quali si trova a vivere e a lavorare cercando, ad un tempo, di comprendere le caratteristiche connotanti la storia e la cultura di quei luoghi e di confrontarsi, di ‘dialogare’ con esse attraverso lo studio e la scrittura”32.

La fisionomia della missione ispettiva è ben delineata dall'autore, negli anni in cui "nessuno pensava alla scuola ed ai maestri, e non erano ancora sorte le associazioni potenti che $[\ldots]$ seppero scuotere l'indifferenza dei governanti e provocare alcune provvide leggi"33:

il legislatore gli aveva conferito una missione tecnica, nel significato più vero e più nobile della parola. Avrebbe dovuto portare fra i banchi della scuola il contributo della sua competenza professionale, sorreggere e correggere gl'insegnanti nell'attuazione dei buoni metodi, orientare

\footnotetext{
${ }^{31}$ B.G. Amorosa, "Il duro calle", cit., p. VI.

${ }^{32}$ G. Palmieri, Berengario Galileo Amorosa. L'intellettuale e lo storico, cit., p. 91.

33 B.G. Amorosa, "Il duro calle", cit.
} 
l'arte didattica verso le nuove teorie della scienza educativa, diffondere ed illustrare fra i suoi dipendenti il pensiero dei pedagogisti ed il progressivo modificarsi ed accrescersi dei programmi, popolarizzare fra le masse l'affetto per la scuola e per essa strappare alle autorità comunali i maggior mezzi possibili ${ }^{34}$.

Ma rispetto a questi alti scopi, il peso di tutto l'apparato burocratico, fatto di incombenze, carte, finche e statistiche interminabili, secondo quanto espone lo stesso Amorosa, aveva sottratto - "nel ciclone della carta e dell'inchiostro" - molta efficacia all'opera tecnica ed educativa dell'ispettore, che nel tempo si era ridotta per lo più a "riferire"35.

A metà strada tra ricordo personale e resoconto, la conoscenza del mondo scolastico è restituita dai tratti realistici di volti e vicende e dalle storie di un osservatore "speciale": ne viene fuori una rappresentazione della scuola tra fine Ottocento e primordi del Novecento che ci trasmette preziose informazioni sulla quotidianità scolastica nei luoghi soprattutto Iontani dai centri principali della vita politica, amministrativa ed educativa.

Vengono riferiti fatti e situazioni concrete sul piano delle applicazioni pratiche delle direttive pedagogico-didattiche: si veda il capitolo su I broccoli e le acacie di S.E. Baccelli, con il "nuovo verbo" con cui nel 1898 si fece "innamorare dei campi le generazioni novelle".

L'ispettore riporta poi notizie sull'andamento delle scuole, informandoci, ad esempio, che una delle scuole rurali visitate era in una "stalla di sei o sette metri quadrati, con relativa greppia ove brucavano dell'erba fresca due pecore: nove o dieci bambini stavano accoccolati per terra. II maestro [...] gridava come un banditore, leggendo una pagina del sillabario che gli scolari ripetevano a coro" ${ }^{36}$. Soffermandosi sulle condizioni dei locali e degli arredi ci viene descritta la scuola che "era posta in una informe casa campestre $[. .$.$] i topi ed i ragni vi erano comodamente istallati e se lo sapevano i poveri$

\footnotetext{
${ }^{34}$ Ivi, p. VII.

${ }^{35}$ B.G. Amorosa, "Il duro calle", cit., p. IX. Amorosa ricorda con una certa amarezza le trasformazioni che avevano condotto l'ispettore "a far lo scribacchino" e come neanche la legge del 24 dicembre 1904 gli avesse restituito l'originaria fisionomia. Le condizioni di carriera e finanziarie non furono del resto migliorate, poiché, soppressa la classe di L. 2000, se ne formarono due di 113 posti ciascuna, fino alla legge sullo stato economico del 30 giugno $1908 \mathrm{n}$. 304. Ibidem.

${ }^{36}$ Ivi, p. 202.
} 
quaderni rosicchiati ed alcuni antichissimi cartelloni a metodo alfabetico tappezzati di ragnatele" ${ }^{37}$. Circa le prassi didattiche appare significativo il capitolo sul Metodo fonico, in cui viene denunciato il "raddoppiamento e rafforzamento [...] quale distinzione accademica di nessun valore".

Ma altri inserti di vita scolastica ci presentano i casi delle maestre, con le loro malattie o le maldicenze di cui erano fatte oggetto, la difficoltà nella scelta dei libri di testo, alcune figure tra gli alunni, oltre che la valutazione delle condizioni degli insegnanti, alle prese con il riconoscimento della loro professione dal punto di vista giuridicoeconomico e sociale, nelle piccole comunità dove erano chiamati a lavorare ed a vivere.

Provenienti da osservatori e narratori dei principali processi di rinnovamento educativo italiano, tra Otto e Novecento, le memorie scolastiche ispettive esemplificate dal volume dell'autore molisano - vanno a connotarsi quale un genere testuale della letteratura scolastica minore certamente meritevole di maggiori attenzioni ed assolutamente da non trascurare. Le attitudini letterarie ed educative rintracciabili in queste pagine manifestano il possesso di una prosa controllata oltre che di un codice espressivo alto, talvolta retorico, sempre fortemente letterario e "carducciano" che si accompagna ad una ricorrente disposizione narrativa incline alla "misura breve" che è possibile estendere, in maniera speculare, alla penna diffusa degli ispettori italiani. La memorialistica ispettiva, si intende cioè evidenziare, attinge ai modelli letterari di fine Ottocento e combina, insieme allo stile in prosa un'esigenza di maggiore rappresentazione realistica dei momenti che hanno segnato l'esperienza individuale nelle vicende collettive della scuola nazionale, così come sono evocati dal racconto. Lungo i capitoli del duro peregrinare "per l'altrui scale" si inanella insieme alla lunga fedeltà ad un modello di letteratura e scrittura trasmesso da una solida erudizione retorico-umanistica, un netto impegno del corpo direttivo e dirigenziale, di segno professionale e dalle alte "idealità educative".

\section{Conclusioni}

\footnotetext{
${ }^{37}$ Ivi, p. 132.
} 
Appare evidente come il dualismo fin qui rimarcato della memoria di scuola quale emerge dalle pagine di Amorosa non segni una distanza tra l'autore e l'ambiente rappresentato ma, al contrario, consenta una lettura attenta e circostanziata delle condizioni della scuola reale, rispetto a quella legale consegnata dalle disposizioni normative e dai programmi, troppo a lungo ritenuta omogenea in tutto il Paese. Soprattutto, dal racconto e dalle vicende narrate viene a galla a tratti netti il ruolo educativo più ampio degli ispettori scolastici i quali, nel loro lungo "tirocinio scientifico e professionale" si mostrano i migliori conoscitori del mondo scolastico e reali artefici dell'opera di unificazione culturale e pedagogica del paese.

In tal senso si può concludere che attraverso opere memorialistiche come quella dell'Amorosa si può ripercorrere il passaggio dalla memoria alla storia. Il "duro calle" prende le mosse da una iniziale forma di memoria individuale dell'ispettore italiano, attenta a restituire in particolare tutto il debito di riconoscenza verso la figura paterna, maestro, che gli ha lasciato in eredità un "patrimonio di onorabilità e dovere"; procede verso la sfera della memoria corporativa professionale, nell'impegno formativo della coscienza di categoria degli ispettori ${ }^{38}$; si spinge fino alla dimensione della memoria collettiva di una rappresentazione di uno spaccato temporale e sociale dell'Italia scolastica otto-novecentesca. Alla memoria personale, cioè, si sommano le memorie plurali della storia comune, nell'ottica delle trasformazioni storiografiche che hanno di recente accentuato il ruolo dell'educazione e della storia della scuola, in rapporto alla vita allargata della società.

Le memorie dell'ispettore molisano, in definitiva, contribuiscono quale genere testuale estensibile ad un'ampia gamma di scritti affini rinvenibili nella letteratura scolastica spesso lasciata in ombra di funzionari e figure dirigenziali nazionali e periferiche, a valutare attraverso quali modi ed esperienze gli ispettori, nell'esercizio tecnico di una funzione altamente sociale, abbiano fattivamente contribuito alla costruzione della visione nazionale della scuola e del maestro.

\footnotetext{
${ }^{38}$ Si ricorda che nella primavera del 1902 fu istituita a Milano l'ANIS, I'Associazione Nazionale fra gli Ispettori Scolastici, sotto la direzione dell'Ispettore centrale Comm. Graziani, vicepresidente, e di Tullio Fontana, segretario. Per le notizie sulla necessità degli ispettori “di organizzarsi per tutelare i propri interessi morali e materiali, specialmente dopo avere assistito e contribuito al risveglio della classe magistrale unitasi in potente organismo atto a richiamare l'attenzione della pubblica opinione e dei governanti sui problemi della scuola", si veda A. Saraz, Vicende storiche ed attuale ordinamento della Direzione ed Ispezione scolastica, cit., p. 127.
} 
Su questo filo il caso di studio proposto ci consegna un tassello significativo di una funzione, quella dell'ispettorato, che specialmente nell'esemplificazione della sua "tecnica" si presenta quale "somma di conoscenze, risultato di esperienze, di osservazioni, di studio, di meditazioni", e di una serie di abilità "acquistate con l'esercizio continuato e l'uso di doti ed attitudini esclusivamente personali"39.

Questa tessera della visione collettiva nazionale del corpo professionale ispettivo comprova, in sintesi, la straordinaria valenza storico-educativa di tale fonte, nell'illuminare le vicende personali ed istituzionali di protagonisti indiscussi di due secoli di vita educativa, in Italia.

\section{Riferimenti bibliografici}

AMOROSA, Berengario Galileo. Autobiografia. In memoria del G. Uff. Dottor Berengario Galileo Amorosa Provveditore agli studi, MDCCCLIV-MCMXXXVII, s.n.t. [1938], pp. 11-23

AMOROSA, Berengario Galileo. Il Molise. Almanacco regionale, Mondadori, 1924, poi ristampato con saggio introduttivo di Giulio DI IORIO, Riccia, Associazione Culturale "P. Vignola", 1990

\footnotetext{
${ }^{39}$ A. Saraz, La tecnica dell'ispezione scolastica, cit., p. 1.
} 
AMOROSA, Berengario Galileo. La scuola in provincia di Terra di Lavoro nei primi tre anni di guerra. Note ed appunti del R. Provveditore agli Studi Dott. B.G. Amorosa, Caserta, Tipografia Economica B. De Simone, 1918

AMOROSA, Berengario Galileo. II “duro calle”. Memorie d'un ispettore scolastico, Milano Roma - Napoli, Società editrice Dante Alighieri di Albrighi \& C., 1912

AMOROSA, Berengario Galileo. Note didattiche e di politica scolastica. Saggi critici, Lanciano, Tip. A. Rosati, 1903

AMOROSA, Berengario Galileo. Riccia nella storia e nel folklore, Casalbordino, Tip. N. De Arcangelis,1903

AMOROSA, Berengario Galileo. Paria moderno. Documenti umani, Palermo, Sandron, 1895

AMOROSA, Berengario Galileo. Cadore. Impressioni liriche, Pieve di Cadore, E. Berengan e C., 1893

AMOROSA, Berengario Galileo. Epodon: versi, Conegliano, Tip. F. Cagnani, 1891

ANTONIETTI, Gaspare. Dal taccuino di un ispettore: noterelle didattiche, Milano, A. Vallardi, 1913, Biblioteca degli ispettori scolastici 2

BARAUSSE, Alberto. Nonostante tanto diluvio di libri scolastici. I libri di testo per le scuole elementari e le indagini ministeriali di Bargoni e Bonghi durante gli anni della Destra storica (1869-1875), Lecce, Pensa Multimedia, 2015

BARAUSSE, Alberto. I maestri all'università: la Scuola pedagogica di Roma 1904-1923, Perugia, Morlacchi, 2004

BARAUSSE, Alberto; D’ALESSIO Michela. “La salvezza della scuola verrà dai tecnici”. La stampa pedagogica in Italia tra idealismo e fascismo: la «Tecnica scolastica» (1923-1927). In HERNANDEZ DIAZ Jose Maria (coord.), La prensa de los escolares y estudiantes. Su contribucion al patrimonio historico educativo, Salamanca, Ediciones Universidad de Salamanca (Collecion Aquilafuente, 210), 2015, pp. 611-626

BARAUSSE, Alberto, D’ALESSIO, Michela. “Dalla piccola alla grande patria”. Libri dialettali e almanacchi regionali per la scuola elementare. In CHIOSSO Giorgio (coord.), TESEO ‘900. Editori scolastico-educativi del primo Novecento, Milano, Editrice Bibliografica, 2008

BANCHESSI, Antonio. Scritti educativi, Milano, A. Vallardi,1920, Biblioteca degli ispettori scolastici 14

BIANCHI, Francesco. Aristide Gabelli nella filosofia positiva e nella pedagogia applicata, Milano, A. Vallardi,1920, Biblioteca degli ispettori scolastici 12 
BORGHESE, Domenico. Lettere a una giovine normalista: (dalle rive della Dora), Milano, A. Vallardi, 1912, Biblioteca degli ispettori scolastici 1

CARLINI, Pietro. Del carattere come formazione psicologica: con l'appendice di una conferenza didattica e due discorsi, Milano, A. Vallardi, 1915, Biblioteca degli ispettori scolastici 7

CAVAZZUTI, Pietro. Il patronato scolastico e le più importanti istituzioni ausiliarie della scuola in Italia, Milano, A. Vallardi,1916, Biblioteca degli ispettori scolastici 9

CHIOSSO, Giorgio. Alfabeti d'Italia. La lotta contro l'ignoranza nell'Italia unita, Torino, SEI, 2011

CIVES, Giacomo. Cento anni di vita scolastica in Italia. Ispezioni e inchieste dall'idealisno a oggi negli scritti di G. Lombardo Radice, G. Isnardi, G. Giovinazzi, F. Betttini, A. Marcucci, L. Volpicelli, L. Borghi, A. Visalberghi, Roma, Armando Editore, 1967

CIVES, Giacomo. Cento anni di vita scolastica in Italia. Ispezioni e inchieste da Gino Capponi a Giuseppe Lombardo Radice, Roma, Armando Editore, 1960

COSSU, Anton Francesco. Note e riflessi di arte didattica, Milano, A. Vallardi,1923, Biblioteca degli ispettori scolastici 16

D’ALESSIO, Michela. Igiene e scuole rurali. Itinerari ed esperienze dell'ANIMI in Basilicata durante il fascismo tra educazione e propaganda. In Atti del Convegno CIRSE Centro Italiano per la Riceraca Storico Educativa, Sguardi della storia. Luoghi, figure, immaginario e teorie dell'educazione (Bologna, 26-27 febbraio 2016). «Rivista di Storia dell'Educazione», v. 3, n. 2 (2016), pp. $43-48$

D'ALESSIO, Michela. Our Schools". The work of the Association of Southern Italy against illiteracy in Basilicata (1921-1928). «History of Education \& Children's Literature», a. X, n. 2 (2015), pp. 463-480

D’ALESSIO, Michela. "Il duro calle”. Memorie di un ispettore scolastico (Berengario Galileo Amorosa, 1865-1937). In YANES, Cristina, MEDA, Juri (coords.), International Symposium, School memories. New trends in Historical research into Education Heuristic Perspectives and Methodological issues, (Siviglia, 22-23 settembre 2015). Abstracts, p. 22

D'ALESSIO, Michela. Life at school: class registers as a new source of studying historical and educational heritage. In BADANELLI RUBIO, Ana M., POVEDA SANZ Maria, RODRIGUEZ GUERRERO Carmen (coords.), Pedagogia museistica. Practicas, usos didacticos e investigacion del patrimonio educativo. Atti della VI Jornadas cientifica della Sociedad Espanola para el Estudio del Patrimonio Historico educativo SEPHE, Madrid, 2224 ottobre 214, Madrid, Universidad Complutense de Madrid, Facultad de Educacion, 2014, pp. 401-409 
D'ALESSIO, Michela. A scuola fra casa e patria. Dialetto e cultura regionale nei libri di testo durante il fascismo, Collana Biblioteca del "Centro di documentazione e ricerca sulla storia delle istituzioni scolastiche, del libro scolastico e della letteratura per l'infanzia" dell'Università degli Studi del Molise, Lecce, Pensa Multimedia, 2013

D’ALESSIO, Michela. Berengario Galileo Amorosa, in CHIOSSO, Giorgio e SANI, Roberto (coords.). DBE - Dizionario Biografico dell'Educazione. 1800-2000, Milano, Editrice Bibliografica, 2013, 2 voll., v. I, scheda n. 75, pp. 45-46

D'ALESSIO, Michela. Vita tra i banchi nell'Italia meridionale. Culture scolastiche in Molise fra Otto e.Novecento, premessa di Alberto BARAUSSE, Collana Biblioteca del "Centro di documentazione e ricerca sulla storia delle istituzioni scolastiche, del libro scolastico e della letteratura per l'infanzia" dell'Università degli Studi del Molise, Campobasso, Palladino Editore, 2011, pp. 13-44

D’ALESSIO, Michela. Scuola e lingua nel Molise di fine Ottocento, Napoli, Edizioni Scientifiche Italiane, 2005

DECOLLANZ, Giuseppe. La funzione ispettiva dalla legge Casati ad oggi, Roma, Armando, 1984

DE MURTAS, Daniele. L'emozione estetica considerata come mezzo di educazione: note di fisio-psicologia, Milano, A. Vallardi, 1915, Biblioteca degli ispettori scolastici 8

DOSSI, Ilario, Pagine di pedagogia di un educatore trentino, Milano, A. Vallardi, 1919, Biblioteca degli ispettori scolastici 10

ESPOSITO, Francesco. La suggestione come mezzo educativo, Milano, A. Vallardi,1914, Biblioteca degli ispettori scolastici 3

MARIANI, Raffaele. Lettere didattiche ad un'esordiente di scuola rurale, Milano, A. Vallardi,1920, Biblioteca degli ispettori scolastici 13

NORSCIA, Vincenzina. Nel campo della pedagogia, Milano, A. Vallardi,1914, Biblioteca degli ispettori scolastici 4

LOMBARDO RADICE, Giuseppe. Presentazione a DAL PIAZ, Riccardo. Esperienze didattiche di un ispettore trentino. Relazione-studio sulle scuole della circoscrizione di Trento (1925-1926), Roma, Associazione per il Mezzogiorno, 1928, p. 3

PALMIERI, Giorgio. Berengario Galileo Amorosa. L'intellettuale e lo storico. In Id., Studi di storiografia molisana, Campobasso, Palladino Editore, 2016, pp. 89-105

PALMIERI, Giorgio. Il contributo della «Rivista abruzzese agli studi del Molise». «Rivista storica del Sannio», a. VIII (2000), n. 13, pp. 305-323 
PALMIERI, Giorgio, SANTORIELLO, Antonio (coords.). Berengario Galileo Amorosa. Atti del Convegno (Riccia, 18 luglio 1987), Riccia, Associazione Culturale "Pasquale Vignola", 1989

PICCIONI, Augusto. Punzecchiature e spunti scolastici, Milano, A. Vallardi,1915, Biblioteca degli ispettori scolastici 6

RENZI, Antonio. “Apostolato”. Memorie autobiografiche di Antonio Renzi, Regio Ispettore scolastico a riposo, Minerbio, Tip. Bevilacqua, [1930]

SANTORIELLO, Antonio. Berengario Galileo Amorosa. In Id. (coord.), Riccia tra biografie e storia. Ricerche su personaggi illustri dell'Ottocento e del Novecento, Riccia, Associazione culturale "Pasquale Vignola”, 1997, pp.196-197

SANTORIELLO, Antonio. I fratelli Amorosa. In Id. (coord.), Riccia tra biografie e storia. Ricerche su personaggi illustri dell'Ottocento e del Novecento, Riccia, Associazione culturale "Pasquale Vignola", 1997, pp. 125-136

SARAZ, Alfredo. Vicende storiche ed attuale ordinamento della Direzione ed Ispezione scolastica, Torino, G.B. Paravia \& C., 1922

SARAZ, Alfredo. La tecnica dell’ispezione scolastica, Torino, G.B. Paravia \& C., 1921

SIMEONI G[s.n.]. Consigli e norme didattiche agli insegnanti esordienti, Milano, A. Vallardi, 1915, Biblioteca degli ispettori scolastici 5

VIGNAO FRAGO, Antonio. Relatos y relaciones autobiograficas de profesores y maestros. In ESCOLANO BENITO, Augustin, HERNANDEZ DIAZ, Jose Maria (coords.), La memoria y el deseo. Cultura de la escuela y educacion deseada, Valencia, Tirant Lo Blanch, 2002, pp. $135-175$. 